\title{
Imagen, localización y evolución de los centros comerciales en Argentina: Un estudio de caso (Buenos Aires)
}

\section{Silvana Sassano Luiz}

Universidad Complutense de Madrid. Departamento de Geografía Humana

silgeo@yahoo.com

\section{Resumen}

Los centros comerciales son los máximos espacios de consumo simbólico y mercantil de la sociedad capitalista actual. El objeto de la investigación de este artículo es analizar los centros comerciales en Argentina, particularmente en la Ciudad Autónoma de Buenos Aires, su evolución, sus características estructurales, su localización geográfica y las motivaciones de los ciudadanos que consumen en ellos. Uno de los principales resultados fue determinar que, en un país semiperiférico, han logrado introducirse y consolidarse como un formato modernizador de la actividad comercial. Por otro lado, se ha demostrado que la concentración espacial de centros comerciales en Buenos Aires está estrechamente relacionada con la alta densidad de población y con el alto poder adquisitivo de la zona donde se emplazan.

Palabras clave: centros comerciales; localización; consumo; Buenos Aires; Argentina.

Resum. Imatge, ubicació i evolució dels centres comercials a l'Argentina: Un estudi de cas (Buenos Aires)

Els centres comercials són els màxims espais de consum simbòlic i mercantil de la societat capitalista actual. L'objecte de la investigació d'aquest article és analitzar els centres comercials a l'Argentina, especialment a la Ciutat Autònoma de Buenos Aires, l'evolució que han seguit, les característiques estructurals que presenten, el lloc on estan situats i les motivacions dels ciutadans que hi consumeixen. Un dels resultats principals fou determinar que, en un país semiperiferic, els centres comercials han aconseguit introduir-s'hi i consolidars'hi com un format de modernització de l'activitat comercial. D'altra banda, s'ha demostrat que la concentració espacial de centres comercials a Buenos Aires està íntimament lligada a l'alta densitat de població i a l'alt poder adquisitiu de la zona on es localitzen.

Paraules clau: centres comercials; localització; consum; Buenos Aires; Argentina. 
Résumé. Image, localisation et évolution des centres commerciaux en Argentine : une étude de cas (Buenos Aires)

Les centres commerciaux sont les espaces les plus grands de la consommation symbolique et du marché de la société capitaliste actuelle. L'objectif de la recherche sur laquelle porte cet article est d'analyser les centres commerciaux en Argentine, et en particulier ceux de la ville autonome de Buenos Aires, leur évolution, leurs caractéristiques structurales, leur localisation géographique et les motivations des citoyens qui y consomment. Les résultats principaux consistent, d'un côté, à montrer comment, dans un pays semipériphérique, les centres commerciaux ont réussi à s'introduire et à se consolider en tant que format modernisant de l'activité commerciale et, d'un autre côté, que la concentration spatiale des centres commerciaux à Buenos Aires est étroitement liée à la densité de la population et au pouvoir d'achat élevé de la zone où ils sont situés.

Mots-clés: centres commerciaux; localisation; consommation; Buenos Aires; Argentine.

Abstract. Image, location and development of malls in Argentina: A case study of Buenos Aires

Malls are the largest spaces of symbolic and mercantile consumption of today's capitalist society. The aim of this article is to analyse the evolution, structural characteristics, geographical location, and citizens' motivations to visit malls in Argentina, particularly in Buenos Aires. The results show how malls have been introduced and consolidated in this semi-peripheral country as a modern form of commercial activity. The spatial location of malls in Buenos Aires is highly and positively related with population density and with the purchasing power of their inhabitants.

Keywords: malls; location; consumption; Buenos Aires; Argentina.

\section{Sumario}

\section{Introducción}

2. El surgimiento y la expansión de los centros comerciales en Argentina

3. Características estructurales de los centros comerciales

4. Los centros comerciales en la Ciudad Autónoma de Buenos Aires
5. Características estructurales de los centros comerciales en la Ciudad Autónoma de Buenos Aires

6. Conclusión

Referencias bibliográficas

\section{Introducción}

En los últimos años, la distribución comercial ha experimentado una transformación y una modernización sustancial que se ve reflejada en el protagonismo que han adquirido los íconos del consumo actual, es decir, los centros comerciales. Son los auténticos protagonistas de la sociedad globalizada de consumo en la que estamos inmersos, las verdaderas catedrales de las mercaderías (Padilla, 2006), las «îles urbaines de la post-modernité» (Ferreira Freitas, 1996) o, como titula su tesis doctoral Pintaudi (1989), "el templo de la mercadería». 
Las grandes ciudades son las receptoras principales de estos cambios a iniciativa de las administraciones locales y, sobre todo, de los sectores empresariales privados, que engendran la «ciudad posmoderna de la imagen, de la diferenciación social y del espectáculo» (Amendola, 2000: 29). El valor simbólico que adquieren los objetos y los espacios (lugares o no lugares) al ser consumidos es primordial, ya sea para lograr un valor agregado en un negocio inmobiliario, por ejemplo, o una diferenciación social entre los propios consumidores. El consumo se convierte en el motor de la vida cotidiana de las familias y de los ciudadanos, que se transforman en consumidores o en ciudadanos imperfectos (Santos, 1987).

Dentro de este nuevo orden, se asiste a una verdadera revolución comercial basada en la creación de simbolos, marcas e imágenes, lo cual da lugar a nuevas formas de estructuración de la ciudad. El valor del producto, su carga simbólica y el espacio en que es consumido implica desigualdades sociales. Estas desigualdades caracterizan a la sociedad urbana contemporánea globalizada y se relacionan con una nueva política cultural, que es abordada por Soja en Thirdspace (1996) y que retoma en Postmetrópolis: «las diferencias entre las personas son creadas de forma intrínseca, externamente impuestas y culturalmente representadas a través de procesos de formación de identidad cargados políticamente» (Soja, 2008: 393).

Por ello, creemos que «la expresión más visible de esta sociedad de consumo - cultura del consumo (Featherstone, 2000) — es la propagación de los grandes centros comerciales» (García Escalona, 1997: 11) en el mundo.

Muchos son los detractores y los críticos de este formato comercial y simbólico y muchos otros los que ven en ellos atractivos y beneficios, pero lo cierto es que se ha convertido en un sector pujante, consolidado, y que ha sido muy bien aceptado por la sociedad actual. Padilla (2006: 25), desde un punto de vista crítico, afirma: «Los desarrolladores y administradores de los shopping centers saben que la realidad del "mundo de fuera" tiene problemas y, en vez de colaborar en la arena política pública para la búsqueda de soluciones con el fin de revalorizar el sentido de la comunidad, escogen la dirección opuesta [...]». Pero, más que reclamar que estos empresarios mejoren la vida pública (que también podrían hacerlo), deberíamos pensar en otro tipo de sociedad y sistema político-económico. Los dueños de los centros comerciales, como todo emprendimiento capitalista, persiguen el máximo beneficio; por ello, no transformarán su esencia capitalista en una obra de caridad urbana y social. Ahora bien, hay que tener claro el discurso que quieren vender a la ciudadanía sobre la idealización de los centros comerciales, la mejor calidad de vida que se respira en su interior, los beneficios de recuperar edificios históricos, las actividades culturales que realizan, etc. Es solo un discurso para atraparnos, hacernos consumir en el propio centro comercial y convertirnos en consumidores en lugar de ciudadanos, como expresaría Santos (1987). No debemos ser tan taxativos y debemos pensar al centro comercial como un posible elemento más del proceso de urbanización (Escudero, 2008). 
En este trabajo, analizaremos los principales indicadores de la expansión del sector en un país semiperiférico ${ }^{1}$ como Argentina (Wallerstein, 1979; Tella, 2007; Lana Seabra y Marvulle Bueno, 2012), particularmente, en la Ciudad Autónoma de Buenos Aires. Describiremos brevemente cómo surgen y se expanden los centros comerciales por el país, analizaremos sus características estructurales, la distribución territorial que presentan y la relación de este crecimiento con las principales motivaciones respecto a su uso.

La investigación considera que las transformaciones por las que atraviesa la Ciudad Autónoma de Buenos Aires desde la década de 1990 son, al mismo tiempo, resultado y, en parte, contribuyen a mantener los procesos de integración, dependencia y globalización económica mundial. Desde este escenario, nos planteamos dos ideas hipótesis básicas:

a) Los centros comerciales son un referente de los habitantes de la Ciudad de Buenos Aires, que han respondido a los parámetros del proceso de globalización, pero, sobre todo, han adquirido características muy localistas, representan un lugar de encuentro e interactúan con el entorno urbano.

b) Los centros comerciales refuerzan la centralidad comercial y urbana, forman parte de la estructura de la ciudad y aún no constituyen el germen de nuevas centralidades, no sustituyen al núcleo principal.

El trabajo de campo y el análisis empírico desarrollados han paliado la escasez de información estadística. La Cámara Argentina de Shopping Centers (CASC) solo facilita cierta información acerca de los centros comerciales asociados a la misma. Ningún otro organismo o publicación registra los datos referentes a la totalidad de los que existen en el país. Respecto a los no asociados a la CASC, intentamos obtener la información directa por medio de entrevista personal, vía Internet, prensa, etc.

Realizamos una aproximación multimétodo, combinando técnicas cuantitativas y cualitativas. La información primaria se obtuvo a partir del levantamiento in situ de las características espaciales y de la observación directa no participativa de todos los centros comerciales de la Ciudad Autónoma de Buenos Aires. Otras fuentes consultadas fueron el Instituto Nacional de Estadísticas y Censos (INDEC), el Gobierno de la Ciudad de Buenos Aires, los periódicos locales de tirada nacional y las páginas web de los centros comerciales.

Para analizar las motivaciones respecto al uso de los centros comerciales, durante el segundo semestre del año 2010, se realizaron 216 entrevistas estructuradas a pie de calle en los alrededores de los centros comerciales de Buenos Aires. La entrevista estructurada permite indagar las motivaciones para consumir en un centro comercial: los más visitados; medios de transporte dispo-

1. El concepto de país semiperiférico fue acuñado por Wallerstein en 1979. Sirve para clasificar a aquellos países que no responden exactamente a la tipología de centro ni de periferia; están en proceso de desarrollo - puesto que son productivos en algunos sectores económicos-, y actúan tanto como periferia para los países centrales, como también como centro para algunas periferias. 
nibles; motivos, antigüedad y frecuencia de la visita; tiempo de permanencia; atributos valorados; percepción socioeconómica (distinción social) respecto a los otros usuarios; secciones a las que se acude con mayor frecuencia (tiendas, hipermercado, cines, área infantil, cafetería), y factores de motivación de la compra. Por lo que respecta a las técnicas cualitativas utilizadas, se realizaron diecinueve entrevistas en profundidad a informantes cualificados: arquitectos responsables de proyectos de centros comerciales como Aldo Volpe (ex representante de Estudio de Arquitectura Juan Carlos López y Asociados), Fernando Galetto (Galerías Pacífico), Eduardo González (Abasto Shopping), Alberto Lípare y Mónica Pérez Arias (gerentes, en distintos períodos, de la Cámara Argentina de Shopping Centers), IRSA, directores y responsables de centros comerciales como Plaza Hudson, Del Parque Shopping, Village Recoleta, Plaza Oeste, Patio Bullrich y Buenos Aires Design, entre otros.

\section{El surgimiento y la expansión de los centros comerciales en Argentina}

Los centros comerciales o shopping centers hacen su aparición en Argentina con bastante retraso en comparación con otros países de la región como Brasil, que abre su primer centro comercial Iguatemi en San Pablo en 1966, o México, con Plaza del Sol en 1969, distantes del Soleil Factory inaugurado en 1987 en el Aglomerado Gran Buenos Aires. Este retraso permitió capitalizar la experiencia externa, principalmente de los EE. UU., según la opinión de Alberto Lípare, ex gerente general de la Cámara Argentina de Shopping Centers (2001). Los cambios en la vida cotidiana a escala global, las nuevas tecnologías, la modernización del sector de la distribución y del consumo, unidos a factores locales como el deterioro de la calle pública, el aumento de la inseguridad y una intención política de insertar a la Argentina dentro del mundo globalizado favorecieron el surgimiento de los shopping centers en este país.

En 1988, aparecen los verdaderos referentes de este formato comercial en el imaginario de los ciudadanos de Buenos Aires, como Patio Bullrich y Spinetto Shopping, en la Ciudad Autónoma de Buenos Aires, y Unicenter, en el Aglomerado Gran Buenos Aires. La década de 1990 fue, sin duda, una etapa de gran expansión, con la apertura del $44,2 \%$ de los centros comerciales. La Ciudad Autónoma de Buenos Aires registró su punto máximo en esta etapa, con un $73,7 \%$, lo que demuestra la gran concentración urbana de los centros comerciales en el país en un primer momento.

En vistas a este crecimiento exponencial, en 1990, se forma la Cámara Argentina de Shopping Centers (CASC), entidad sin fines de lucro que agrupa y ordena la actividad del sector (Pérez Arias, 2007; CASC, 2014). Es miembro del International Council of Shopping Centers (ICSC), asociación mundial sin fines de lucro fundada en 1957.

Luego de una etapa de estancamiento y paralización de la actividad comercial, producto de la recesión y de la crisis general, Argentina experimenta, desde 2004, un gran crecimiento económico: se recuperan el empleo y los ingresos de la población, lo cual, sumado a la entrada de turismo internacional por los 
beneficios cambiarios y los bajos precios del país, genera una alza del consumo propicio para nuevos desarrollos comerciales. En las ediciones cuarta y quinta del Congreso Internacional de Shopping Centers, celebradas en septiembre de 2011 y de 2013, respectivamente, se destacó el buen momento que atraviesa el sector, pese a la crisis económica mundial de los últimos años (Blaksley, 2011; Osuna Gutiérrez, 2013). Los proyectos paralizados se hacen efectivos y se suman otros nuevos, que se localizan, fundamentalmente, en ciudades del interior del país y en los partidos ${ }^{2}$ del Aglomerado Gran Buenos Aires. Desde 2004 hasta mayo de 2014, se han abierto cincuenta y cinco centros comerciales en todo el país. En la Ciudad Autónoma de Buenos Aires, solo se registran tres nuevas aperturas en este mismo período, aunque, en 2009, se construye el centro comercial más grande de la ciudad: Dot Baires Shopping.

\section{Características estructurales de los centros comerciales}

En veinticinco años de historia, se han abierto 113 centros comerciales en Argentina. El 16,81\% se localizan en la Ciudad Autónoma de Buenos Aires; el 33,6\%, en los partidos del Aglomerado Gran Buenos Aires, y el 49,5\%, en el resto del país.

Hay una gran centralización en el Aglomerado Gran Buenos Aires: en $2.589 \mathrm{~km}^{2}$ (un $0,093 \%$ de su superficie), se concentran cincuenta y siete centros comerciales, el 50,4\% del total; por el contrario, en el 99,9\% de la superficie restante, se distribuyen cincuenta y seis centros comerciales, el 49,5\%. Esta concentración espacial está determinada por la densidad de población, ya que el 30,86\% de los habitantes del país se localiza en el Aglomerado Gran Buenos Aires.

Sin incluir el Aglomerado Gran Buenos Aires, los centros comerciales están localizados en las provincias más densamente pobladas, como Córdoba (3.066.801 habitantes), Santa Fe (3.000.701 habitantes) y Mendoza (1.579.651 habitantes). En general, en ciudades con más volumen demográfico y/o con mayor nivel socioeconómico.

Tabla 1. Comparación de la distribución de centros comerciales, superficie y población por regiones (mayo de 2014)

\begin{tabular}{|c|c|c|c|c|c|c|c|c|}
\hline & $\begin{array}{c}\text { Ciudad } \\
\text { Autónoma de } \\
\text { Buenos Aires }\end{array}$ & $\%$ & $\begin{array}{c}\text { Partidos del } \\
\text { Aglomerado } \\
\text { Gran Buenos } \\
\text { Aires }\end{array}$ & $\%$ & $\begin{array}{l}\text { Resto del país } \\
\text { (sin Aglomerado } \\
\text { Gran Buenos } \\
\text { Aires) }\end{array}$ & $\%$ & Total país & $\%$ \\
\hline Centros comerciales & 19 & 16,81 & 38 & 33,63 & 56 & 49,56 & 113 & 100 \\
\hline Superficie $\left(\mathrm{km}^{2}\right)$ & 203 & 0,01 & 2.386 & 0,09 & 2.778 .014 & 99,91 & 2.780 .603 & 100 \\
\hline Población & 2.776 .138 & 7,11 & 9.270 .661 & 23,75 & 26.989 .469 & 69,14 & 39.036 .268 & 100 \\
\hline
\end{tabular}

Fuente: elaboración de la autora en base a datos propios y de la Cámara Argentina de Shopping Centers.

2. El partido es una división administrativa local propia de la provincia de Buenos Aires, en Argentina (nota del editor). 


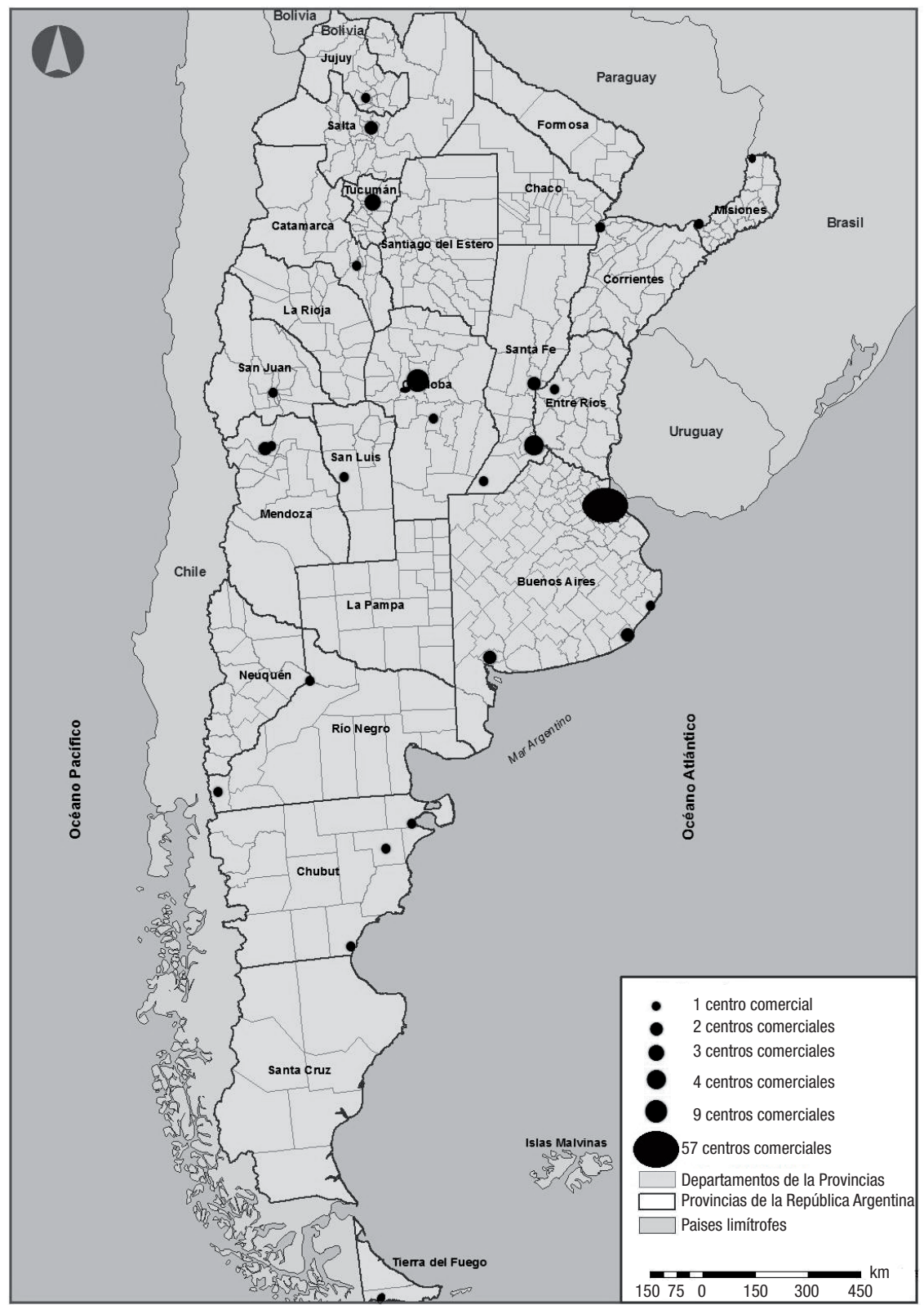

Figura 1. Localización de centros comerciales en Argentina (mayo de 2014).

Fuente: elaboración de la autora en base a datos propios y de la Cámara Argentina de Shopping Centers. 
En los partidos del Aglomerado Gran Buenos Aires, el 50\% de los centros comerciales se concentran en la zona norte (partidos de Pilar, San Isidro, Tigre, Escobar y Vicente López); el 30\%, en la zona sur (partidos de Avellaneda, Quilmes, Lomas de Zamora, Adrogué y Cañuelas), y el 20\%, en la zona oeste (partidos de Morón, San Martín, Moreno y La Matanza). Esta distribución responde fundamentalmente al criterio de densidad de población y de nivel socioeconómico; la región norte concentra el mayor número de centros comerciales localizados en zonas de poder adquisitivo alto y medio alto, especialmente en el partido de Pilar, los cuales abastecen a los barrios privados, countries clubs y condominios cerrados propios del poblamiento urbano de la zona. Estas nuevas formas residenciales privadas y los centros comerciales protagonizan los grandes cambios en el mercado del suelo ciudadano, lo cual genera nuevas centralidades urbanas (Tella, 2005). La zona oeste y sur, en cambio, con un nivel socioeconómico menor, dispone de varios centros comerciales tipo factory (a precios de fábrica) y tipo galería comercial, normalmente con un hipermercado como locomotora.

La misma concentración espacial se aprecia en la superficie bruta alquilable (SBA). Para desarrollar este punto, se han analizado datos parciales, al no disponer de la totalidad de ellos ${ }^{3}$. La SBA total en mayo de 2014 es de $1.966 .826,51 \mathrm{~m}^{2}$.

Si analizamos la SBA creada por períodos y regiones (tabla 2), se confirma la relación entre las aperturas de centros comerciales y el crecimiento de aquella. Desde 1990 hasta 2001, la SBA creada alcanzó la cota histórica máxima

Tabla 2. Evolución de la SBA $\left(\mathrm{m}^{2}\right)$ por período de apertura y regiones (mayo de 2014)

\begin{tabular}{|c|c|c|c|c|c|c|c|c|}
\hline \multirow[b]{2}{*}{ Periodo } & \multicolumn{2}{|c|}{$\begin{array}{l}\text { Ciudad Autónoma } \\
\text { de Buenos Aires }\end{array}$} & \multicolumn{2}{|c|}{$\begin{array}{c}\text { Partidos del } \\
\text { Aglomerado G. B. A. }\end{array}$} & \multicolumn{2}{|c|}{ Resto del país } & \multicolumn{2}{|c|}{ Total } \\
\hline & $\mathrm{N}^{\circ}$ C. C. & $\mathrm{SBA}\left(\mathrm{m}^{2}\right)$ & No C. C. $^{*}$ & $\mathrm{SBA}\left(\mathrm{m}^{2}\right)$ & № C. C. & $\mathrm{SBA}\left(\mathrm{m}^{2}\right)$ & $\mathrm{N}^{\circ} \mathrm{C} . \mathrm{C}$. & $\mathrm{SBA}\left(\mathrm{m}^{2}\right)$ \\
\hline $\begin{array}{l}\text { Hasta } 1 \text { de enero } \\
\text { de } 1990\end{array}$ & 2 & $26.662,36$ & 3 & $131.412,44$ & 0 & 0 & 5 & $158.074,80$ \\
\hline 1990-2001 & 14 & $291.238,99$ & 16 & $536.564,40$ & $18^{\star}(20)$ & $369.672,36$ & $48^{\star}(50)$ & $1.197 .475,75$ \\
\hline 2002-2003 & 0 & 0 & 0 & 0 & $1^{\star}(3)$ & 4.100 & $1^{*}(3)$ & 4.100 \\
\hline $\begin{array}{l}\text { Desde } 2004 \text { hasta } \\
1 \text { de mayo de } 2014\end{array}$ & 3 & $83.951,00$ & $11^{*}(19)$ & $210.670,00$ & $17^{\star}(33)$ & $312.554,96$ & $31^{\star}(55)$ & $607.175,96$ \\
\hline Total & 19 & $401.852,35$ & $30^{*}(38)$ & $878.646,84$ & $36^{\star}(56)$ & $686.327,32$ & $85^{\star}(113)$ & $1.966 .826,51$ \\
\hline
\end{tabular}

* Según los datos disponibles.

C. C.: centros comerciales.

Entre paréntesis: total de C. C. en ese período.

Fuente: elaboración de la autora en base a datos propios y de la Cámara Argentina de Shopping Centers.

3. Como hemos mencionado en la introducción, la Cámara Argentina de Shopping Centers solo facilita algunos datos de los centros comerciales asociados a la misma. No se registran en ningún otro organismo o publicación los datos referentes a la totalidad de centros comerciales del país. 


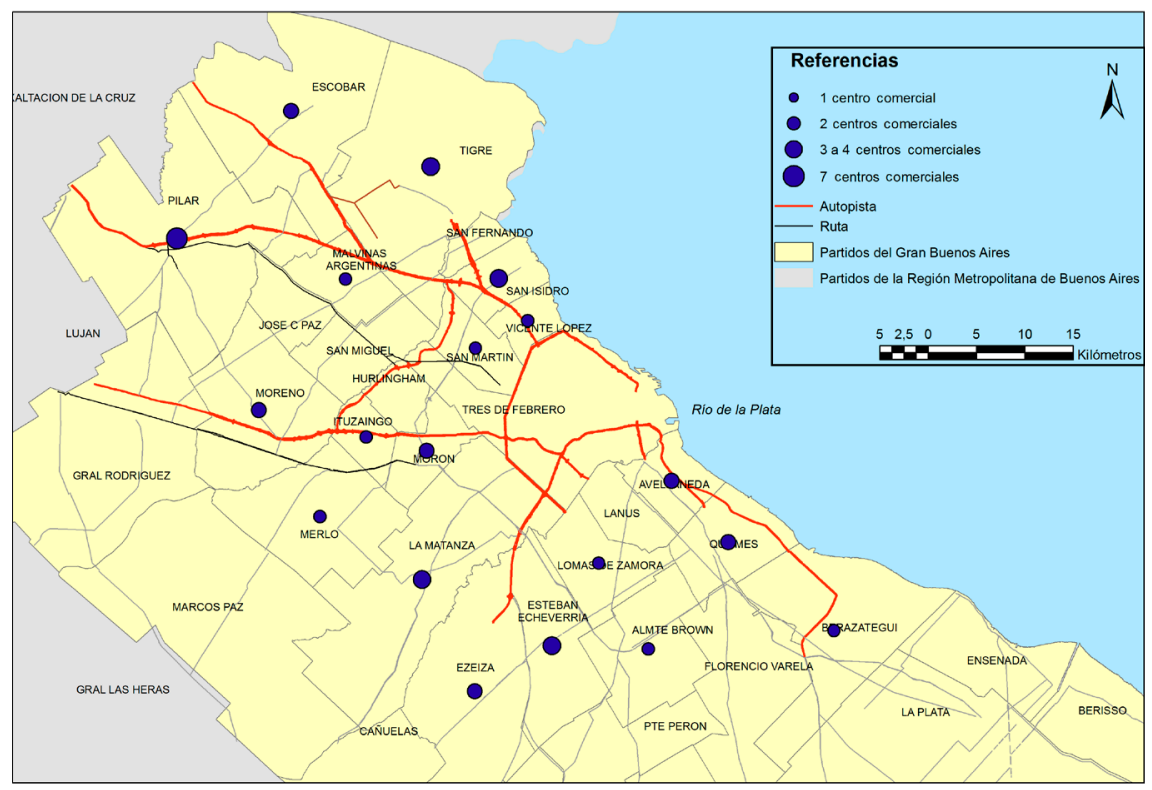

Figura 2. Localización de centros comerciales en los partidos del Aglomerado Gran Buenos Aires (mayo de 2014).

Fuente: elaboración de la autora en base a datos propios y de la Cámara Argentina de Shopping Centers.

hasta la actualidad, con 1.197.475,75 $\mathrm{m}^{2}$, muy superior a la del último período, con $607.175,96 \mathrm{~m}^{2}$ de SBA construidos y 55 centros comerciales abiertos (aunque se dispone de información solo de 31). Por regiones, los partidos del Aglomerado Gran Buenos Aires concentran el 44,6\% de la SBA total, seguido del resto del país, con el 34,9\%, y, finalmente, la Ciudad Autónoma de Buenos Aires, con un 20,4\%. El porcentaje del resto del país ha crecido sustancialmente en los últimos años, por la apertura de centros comerciales en ciudades más pequeñas y que no habían sido conquistadas todavía por este formato comercial. El potencial de desarrollo de nuevos centros comerciales en el resto del país durante los próximos años es muy grande. Hay una gran cantidad de proyectos en marcha por parte de diversos desarrolladores.

En cambio, si analizamos la densidad comercial ( $\mathrm{m}^{2} \mathrm{SBA} / 1.000$ habitantes), es mucho mayor en la Ciudad Autónoma de Buenos Aires respecto a los partidos del Aglomerado y aún más en comparación con el resto del país. La media nacional es de 50,38 $\mathrm{m}^{2} \mathrm{SBA} / 1.000$ habitantes, cifra casi tres veces inferior a la de la ciudad, con 144,7 $\mathrm{m}^{2} \mathrm{SBA} / 1.000$ habitantes, casi dos veces inferior a los partidos del Aglomerado Gran Buenos Aires y, en cambio, muy por debajo el resto del país, con una densidad comercial de $25,4 \mathrm{~m}^{2} \mathrm{SBA} / 1.000$ habitantes (tabla 3). Esto determina la gran concentración comercial en la Ciudad 
Tabla 3. Distribución de centros comerciales y de SBA, densidad comercial ( $m^{2}$ SBA $/ 1.000$ habitantes) y dimensión media por regiones (mayo de 2014)

\begin{tabular}{lcccccc}
\hline & C. C. ${ }^{*}$ & $\mathrm{SBA}\left(\mathrm{m}^{2}\right)$ & $\%$ & Población & $\mathrm{m}^{2} \mathrm{SBA} / 1.000$ hab. & Dimensión media \\
\hline $\begin{array}{l}\text { Ciudad Autónoma } \\
\text { de Buenos Aires }\end{array}$ & 19 & $401.852,35$ & 20,43 & 2.776 .138 & 144,75 & $21.150,12$ \\
$\begin{array}{l}\text { Partidos del } \\
\text { Aglomerado G. B. A. }\end{array}$ & 30 & $878.646,84$ & 44,67 & 9.270 .661 & 94,78 & $29.288,23$ \\
Resto del país & 36 & $686.327,32$ & 34,90 & 26.989 .469 & 25,43 & $19.064,65$ \\
Total & 85 & $1.966 .826,51$ & 100,00 & 39.036 .268 & 50,38 & $23.139,14$ \\
\hline
\end{tabular}

* De los datos disponibles.

Fuente: elaboración de la autora en base a datos propios y de la Cámara Argentina de Shopping Centers.

Autónoma de Buenos Aires y también si consideramos al Aglomerado Gran Buenos Aires en su conjunto.

Esta elevada densidad comercial apoya la tesis de la bastante mayor localización en trama urbana muy consolidada respecto a la suburbana. Sin embargo, esta tendencia podría modificarse en los próximos años debido al incremento de la SBA y de aperturas en los suburbios del Gran Buenos Aires y en el resto del país.

La evolución de la dimensión media de la SBA de los centros comerciales no ha sido lineal. Hasta el inicio de 1990, la dimensión media fue la más elevada, con 31.614,96 m². En el segundo período, 1990-2001, la dimensión media desciende a $24.947,4 \mathrm{~m}^{2}$, y lo hace después a valores prácticamente inexistentes durante el período de crisis económica (2001-2003). En la última etapa comprendida entre 2004 y la actualidad, se recupera sensiblemente hasta $19.586,3 \mathrm{~m}^{2}$. La densidad media está disminuyendo por la apertura de centros comerciales de menores dimensiones y más diversificados.

Según los datos disponibles, el mayor porcentaje de plazas de aparcamiento se localiza en los partidos del Aglomerado Gran Buenos Aires, con el 52,7\% (50.136 plazas), seguido del resto del país, con el 31,9\% (30.387 plazas). La Ciudad Autónoma de Buenos Aires es la región con el menor número de plazas de aparcamiento $(15,2 \%, 14.451)$; primero, por disponer de menos centros comerciales; segundo, porque, al estar dentro de la trama urbana compacta, no dispone de suelo vacío, salvo en forma subterránea, y tercero, porque se accede fácilmente en transporte público o a pie, por su localización urbana central. La ciudad central está recorrida por cinco líneas de metro, dos de premetro ${ }^{4}$, siete de trenes que continúan hacia los partidos del Aglomerado Gran Buenos Aires y ciento veinticuatro líneas de autobuses (colectivos). Salvo Parque Brown Factory Outlet, localizado en Villa Lugano, en la periferia sur de la ciudad, donde el modo de acceso es relativamente deficitario, con el premetro y cuatro líneas de autobuses, el resto de centros comerciales de la Ciudad Autónoma

4. El premetro es un tipo de metro ligero al aire libre. 
Tabla 4. Centros comerciales con salas de cine por región (mayo de 2014)

\begin{tabular}{lccccc}
\hline & C. C. * & Distribución \% & C. C. c/ salas cine & Distribución \% & \% C. C. con cine / total C. C. \\
\hline $\begin{array}{l}\text { Ciudad Autónoma } \\
\text { de Buenos Aires }\end{array}$ & 19 & 20,00 & 10 & 17,86 & 52,63 \\
$\begin{array}{l}\text { Partidos del } \\
\text { Aglomerado G. B. A. }\end{array}$ & 33 & 34,77 & 18 & 32,14 & 54,55 \\
Resto del país & 43 & 45,26 & 28 & 50,00 & 65,12 \\
Total & 95 & 100,00 & 56 & 100,00 & 58,95 \\
\hline
\end{tabular}

* De los datos disponibles.

Fuente: elaboración de la autora en base a datos propios y de la Cámara Argentina de Shopping Centers.

Tabla 5. Volumen mensual total y promedio de visitantes por regiones (mayo de 2014)

\begin{tabular}{lccccc}
\hline & C. C. * & \multicolumn{2}{c}{ Distribución \% Volumen de visitantes } & Distribución \% & Visitantes por C. C. \\
\hline $\begin{array}{l}\text { Ciudad Autónoma de } \\
\text { Buenos Aires }\end{array}$ & 19 & 23,08 & 11.240 .000 & 26,29 & 591.579 \\
$\begin{array}{l}\text { Partidos del Aglome- } \\
\text { rado G. B. A. }\end{array}$ & 27 & 34,62 & 16.343 .500 & 38,23 & 605.315 \\
Resto del país & 33 & 42,31 & 15.166 .000 & 35,48 & 459.576 \\
Total & 79 & 100,00 & 42.749 .500 & 100,00 & 548.071 \\
\hline
\end{tabular}

* De los datos disponibles.

Fuente: elaboración de la autora en base a datos propios y de la Cámara Argentina de Shopping Centers.

están servidos por una amplia red de autobuses, en promedio unas 10 líneas por cada centro. Por metro, se accede al $36 \%$ de los centros comerciales y por tren, al $15 \%$.

El 58,9\% de centros comerciales tiene salas de cine, lo que demuestra la importancia de este sector en la sociedad de consumo actual, por ello actúan como una locomotora en muchos de los casos. Por regiones, el 52,6\% de los centros comerciales en la Ciudad Autónoma de Buenos Aires dispone de salas de cine, el 54\% en los partidos del Aglomerado, y sustancialmente más en el resto del país, con un 65\% (tabla 4). Históricamente, Buenos Aires ha tenido una alta oferta cultural (teatros, cines, librerías, cafeterías literarias, etc.) en comparación con las otras regiones.

Mensualmente, los centros comerciales argentinos reciben un total de 42.749.500 visitantes. Por regiones, los de los partidos del Aglomerado Gran Buenos Aires han recibido una mayor afluencia, con el 38,2\%, seguido del resto del país, con un 35,4\%, y la Ciudad Autónoma de Buenos Aires, con un 26,3\%. Este comportamiento es lógico, ya que la Ciudad de Buenos Aires y los partidos del Aglomerado Gran Buenos Aires abarcan el 0,10\% de la superficie total del país y concentran el 30\% de la población total. Los centros comerciales del Aglomerado disponen de buenas vías de acceso y los visitantes se desplazan hasta ellos en vehículos propios, motivados por las compras y el ocio (cines, juegos infantiles, restauración). 
En síntesis, desde las primeras aperturas, se logra, en pocos años, una expansión muy acelerada de centros comerciales y de superficie construida. La desaceleración económica de fines de la década de 1990 paraliza la actividad del sector. Con la estabilización económica y política lograda unos años más tarde, poco a poco se va recuperando la actividad comercial, la capacidad de consumo de la sociedad argentina y la inauguración de nuevos centros comerciales.

\section{Los centros comerciales en la Ciudad Autónoma de Buenos Aires}

El 16,81\% de los centros comerciales del país se localizan en la Ciudad Autónoma de Buenos Aires. La ciudad está dividida en 48 unidades territoriales llamadas «barrios», de los que solo doce poseen centros comerciales. Su distribución responde, primordialmente, al nivel socioeconómico del área circundante donde se emplazan.

En la Ciudad Autónoma de Buenos Aires, la dinámica demográfica se ve reflejada por zonas con comportamientos disímiles. Por razones históricas, económicas o geográficas, las familias de mayores recursos económicos se ubican en las zonas donde el valor de la tierra es mayor y viceversa. «Históricamente, hay barrios donde se ubican estratos sociales con mayor poder adquisitivo, más

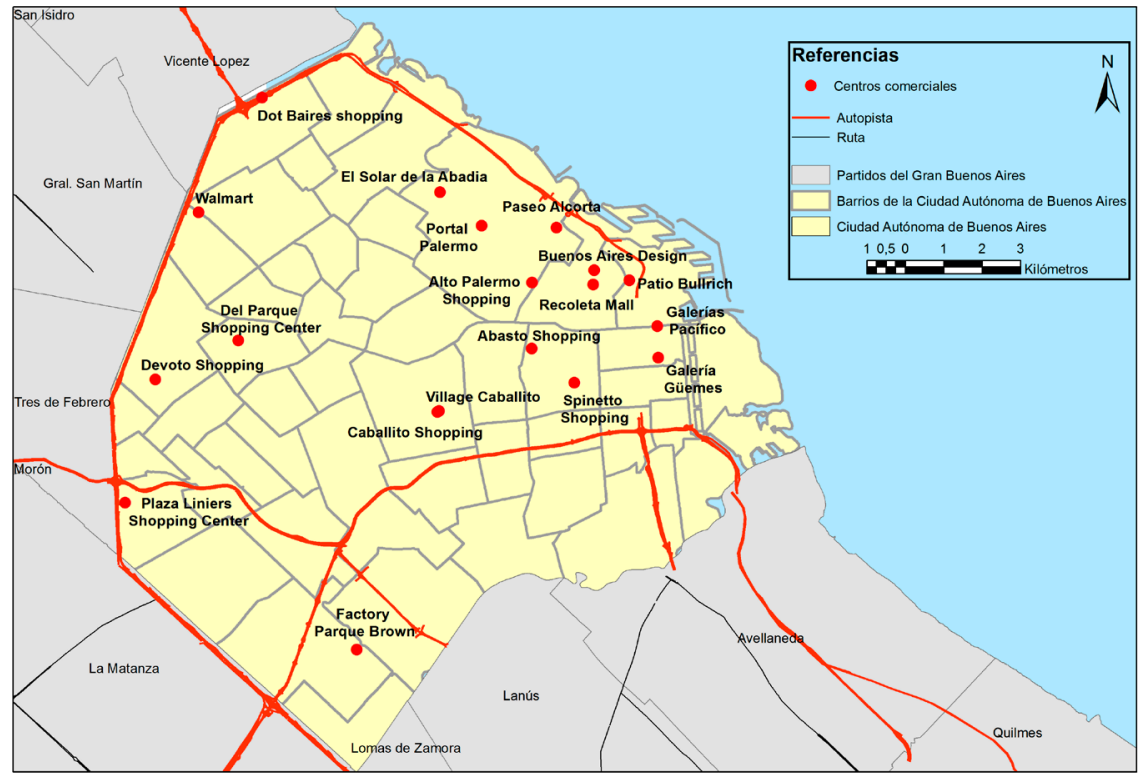

Figura 3. Localización de centros comerciales en la Ciudad Autónoma de Buenos Aires (mayo de 2014).

Fuente: elaboración de la autora en base a datos propios y de la Cámara Argentina de Shopping Centers. 
allá de los precios actuales que sirven como referencia» (Equipo de Gestión Económica y Social, Ciudad Autónoma de Buenos Aires, 2009). Para hacer un análisis estricto y determinar el nivel socioeconómico por zonas, hay que tener en cuenta una serie de variables económicas, sociales, geográficas e históricas, lo que escapa al análisis de este artículo. Pero el indicador más utilizado en Argentina, que se elabora desde hace 40 años en base a los datos del Censo Nacional de Población, Hogares y Vivienda del Instituto Nacional de Estadísticas y Censos (INDEC), es el de Necesidades Básicas Insatisfechas (NBI). Este método consiste en verificar si los hogares han satisfecho una serie de necesidades como el acceso a una vivienda adecuada, la disponibilidad de agua y la posibilidad de gozar de servicios sanitarios fundamentales, la educación básica y la capacidad económica, considerando pobres a aquellos que no lo han logrado (Equipo de Gestión Económica y Social, Ciudad Autónoma de Buenos Aires, 2009).

El mayor porcentaje de población con NBI se localiza en el sur de la ciudad (figura 4), en los barrios de Villa Soldati, Villa Lugano, Nueva Pompeya,

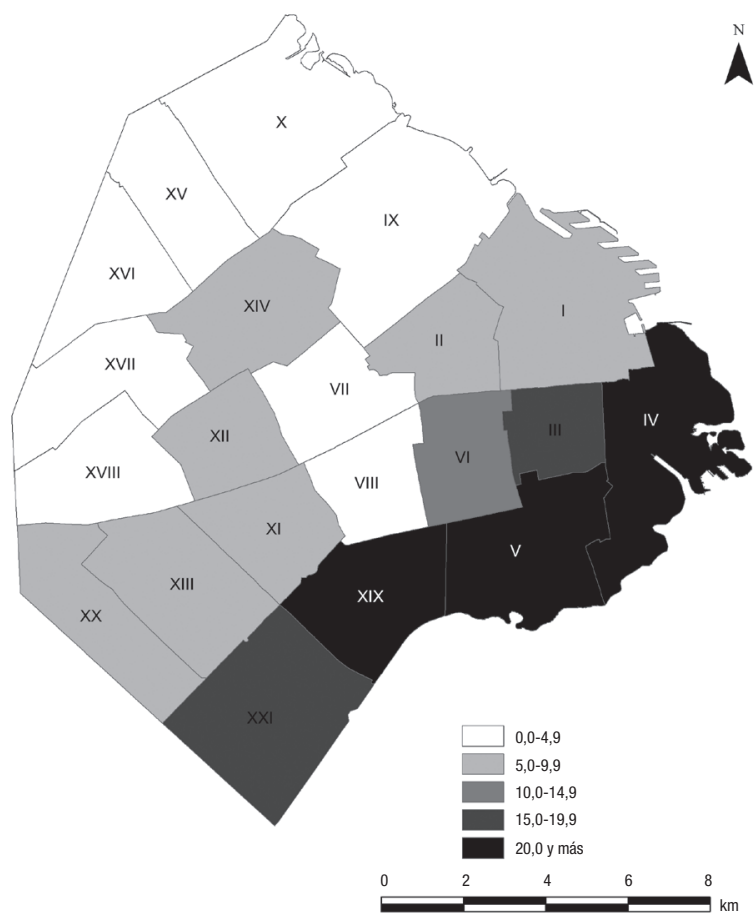

Figura 4. Porcentaje de población en hogares con NBI por distrito escolar (Ciudad Autónoma de Buenos Aires, 2001).

Fuente: Dirección General de Estadística y Censos (Ministerio de Hacienda GCBA) sobre la base de datos de INDEC (Censo Nacional de Población y Vivienda, 1991, y Censo Nacional de Población, Hogares y Vivienda, 2001). 
Barracas, La Boca y Monserrat (distritos escolares XIX, XXI, IV y V), mientras que, en el norte, en barrios como Belgrano, Palermo y Núñez (IX, X) y algunos del oeste de la ciudad, como Saavedra, Villa Urquiza y Devoto (X, XV y XVII), muestran unos valores de NBI menores. Hay que hacer la salvedad, por ejemplo, en el distrito escolar I, con un 7,1\% de NBI, que, si bien abarca el barrio de Recoleta, considerado, junto a Puerto Madero, los de mayor nivel socioeconómico de la ciudad, también se corresponde con los barrios de Retiro, San Nicolás y parte de Balvanera, donde se localiza la villa miseria ${ }^{5}$ de Retiro, villas 31 y 31 bis, una de las más grandes de la ciudad.

La zona norte concentra la mayor cantidad de centros comerciales que se encuentran en la ciudad, exactamente el 38,8\% del total, en los barrios de Retiro, Palermo, Recoleta y Saavedra, zona de alto poder adquisitivo, muy bajo NBI y elevada densidad poblacional; las zonas centro y oeste concentran, cada una, el 22,2\% en los barrios de Caballito y Balvanera y Villa Devoto, Villa del Parque, Liniers y Villa Pueyrredón. En la zona este, en medio del casco histórico urbano y sobre la tradicional calle Florida, aparecen dos centros comerciales emblemáticos, como Galerías Güemes y Galería Pacífico, y, finalmente, en la zona sur, está el Parque Brown Factory Outlet. Este último centro responde a las características tipológicas de galería comercial a precios de fábrica, con una locomotora como el hipermercado Jumbo, y está localizado en un barrio de bajo poder adquisitivo y alto porcentaje de NBI.

$\mathrm{Al}$ año siguiente de la apertura del primer centro comercial del país, en pleno casco urbano de la Ciudad Autónoma, se inauguran Patio Bullrich y Spinetto, ambos, resultado de un proceso de rehabilitación de edificios históricos de la capital.

La década de 1990 representó el máximo esplendor de este nuevo formato (Leston, 1992), por la apertura de catorce centros comerciales, de tipología, localización, etc. variadas. En 1990, aparece el referente de shopping center en la ciudad, Alto Palermo Shopping, con una escala mayor y con la particularidad arquitectónica de haber sido construido enlazándose en la trama urbana existente, y no como una caja ciega, con muros ciegos apenas maquillados o un espacio sin relación con el entorno (Sarlo, 2000, 1996; Grossman, 1988). Por un acuerdo con la municipalidad, se debió abrir la calle Arenales, que forma parte del proyecto de construcción del centro comercial, pasando por debajo del mismo. En ese mismo año, también como resultado de la rehabilitación de un viejo mercado, abre sus puertas Plaza Liniers, un centro comercial barrial localizado en la periferia de la Ciudad Autónoma, en el barrio de Liniers, zona de gran densidad comercial y alto flujo de personas, al ser un lugar de intercambio y concentración de transporte público.

En 1992, con formatos totalmente disímiles y orientados a sectores sociales también diferentes, abren sus puertas Paseo Alcorta, Parque Brown Factory

5. «Villa miseria» es el nombre que se da, en Argentina, a los asentamientos informales, precarios. 
Outlet y Galerías Pacífico, este último producto de la rehabilitación del edificio histórico del mismo nombre. Se localizan en Palermo, Villa Lugano y San Nicolás, respectivamente, barrios caracterizados por indicadores socioeconómicos contrapuestos, como son los casos de Palermo y Villa Lugano. Paseo Alcorta es un edificio de nueva construcción, de altas calidades y diseño arquitectónico refinado, especializado en las últimas tendencias de la moda. Algo que llama la atención es la presencia de su locomotora, el hipermercado Carrefour, que, si bien se encuentra anexo al centro comercial, también forma parte del mismo. En cambio, Parque Brown Factory Outlet es una galería comercial asociada al hipermercado Jumbo y a Easy, un home center. Arquitectónicamente, no tiene tanta relevancia, se parece más a una nave industrial que a un lujoso centro comercial. Galerías Pacífico, en pleno casco histórico de la ciudad y sobre la tradicional calle peatonal Florida, atrae a visitantes que transitan por la zona y a numerosos turistas extranjeros.

En 1993, se inaugura el primer centro comercial temático en el barrio de Recoleta (Buenos Aires Design), orientado al diseño, a la construcción, al equipamiento y a la decoración, con marcas exclusivas y donde se exponen las últimas tendencias del sector. En los años 1994 y 1995, tres centros comerciales barriales como Caballito Shopping Center, Del Parque Shopping y El Solar de la Abadía, en los barrios de Caballito, Villa del Parque y Palermo, respectivamente, nacen para satisfacer la demanda local. Sus dimensiones son menores, lo que no significa que el diseño y las calidades arquitectónicas también lo sean, muy al contrario. El Solar de la Abadía es producto de la rehabilitación de una antigua fábrica de hielo. El edificio que hoy ocupa Caballito Shopping Center había pertenecido a las tiendas del Hogar Obrero, y Del Parque Shopping es de nueva construcción. Similares características posee el Devoto Shopping, abierto en plena crisis económica de 2001.

Portal Palermo y Walmart comienzan su actividad en 1996, en los barrios de Palermo y Villa Pueyrredón, respectivamente. Se trata de galerías comerciales asociadas al hipermercado Jumbo, en el primer caso, y Walmart, en el segundo. Son de nueva construcción y presentan características arquitectónicas similares a Parque Brown Factory Outlet.

Abasto Shopping, inaugurado en 1998, había sido el gran gigante de la ciudad, hasta que lo desplazó Dot Baires Shopping en mayo de 2009. Abasto Shopping nace de la rehabilitación de un viejo mercado histórico de la ciudad, en el marco de un megaproyecto de transformación urbana en el barrio de Balvanera (Sassano, 2001).

En julio de 1999, en pleno barrio de Recoleta, aparece Village Recoleta (hoy Recoleta Mall), un centro comercial temático dedicado íntegramente al cine con algunas tiendas comerciales y de restauración como complemento. Es un lujoso edificio con altas calidades interiores, muy colorido y con un diseño arquitectónico de primer nivel (Baum, 2007). En 2007, cambia de propietarios y reabre sus puertas el 14 de junio de 2011 con el nombre de Recoleta Mall. Se mantiene la estructura general, el diseño y el lujo, pero se amplía la oferta comercial y gastronómica. 
La crisis de diciembre de 2001 paralizó la expansión de los centros comerciales en la Ciudad Autónoma de Buenos Aires. En la entrevista mantenida con Eduardo Goilenberg (2002), nos comentaba lo mucho que había afectado la crisis económica al sector:

[...] el shopping no es un hipermercado, no son compras de primera necesidad [...]. Las marcas dejaron de vender tanto y llegó diciembre (2001) y fue el caos. Diciembre y enero... te diría que vivimos una tormenta hasta marzo..., muy complicada por varios motivos: primero, por el ánimo de la gente, porque el que no tiene ánimo no tiene ganas de comprar; segundo, porque no había dinero en el bolsillo; tercero, porque se acabaron las tarjetas de crédito en cuotas y fue terrible cómo esto afectó a la venta en los shoppings. [...] se hicieron rebajas a los alquileres, tratamos de ir acompañando a la crisis y hoy te diría que estamos de nuevo con los alquileres a full.

En 2005, abre tímidamente sus puertas Village Caballito, en el barrio de Caballito, con la misma orientación y diseño arquitectónico que el Recoleta Mall, aunque con distintos propietarios. Galería Güemes adquiere el rótulo de centro comercial en estos años, y si bien consideramos la fecha de apertura de esta última en 2005, en realidad, es una antigua galería comercial de 1918 rehabilitada. Cuatro años más tarde, en mayo de 2009, abre el centro comercial más grande de la ciudad, Dot Baires Shopping, en el barrio de Saavedra, próximo a dos vías de circulación rápida: la avenida Panamericana y la avenida del General Paz.

Por tanto, el 78\% de las aperturas de los centros comerciales en la Ciudad Autónoma de Buenos Aires se concentró en los años noventa, década también de grandes transformaciones urbanas e inversiones inmobiliarias. La crisis económica de 2001-2002 paralizó todos los proyectos que estaban por desarrollarse en la ciudad. Veremos si, en un futuro próximo y con la relativa «estabilidad» económica y política actual, se vuelve a invertir en el sector como en años anteriores, aunque sí podemos confirmar que con más lentitud. Las aperturas de centros comerciales se están registrando en los partidos del Gran Buenos Aires y el resto del país, en ciudades de menor tamaño y con un nicho comercial aún por explotar.

\section{Características estructurales de los centros comerciales en la Ciudad Autónoma de Buenos Aires}

En mayo de 2014, la SBA en los centros comerciales de la Ciudad Autónoma alcanzaba los 401.852,35 $\mathrm{m}^{2}$, de los cuales 291.238,99 fueron creados durante la década de 1990. Los dos barrios con mayor SBA son Palermo, con 111.329, $16 \mathrm{~m}^{2}$ distribuidos en cuatro centros comerciales, y el barrio de Saavedra, con $76.600 \mathrm{~m}^{2}$ en un único centro comercial, Dot Baires Shopping. En Balvanera, se localizan dos centros comerciales cuyos edificios fueron antiguos mercados de abastos del siglo pasado, Abasto de Buenos Aires y Spinetto Shopping. Los barrios con menor SBA son Liniers y Villa del Parque, con 5.800 y 


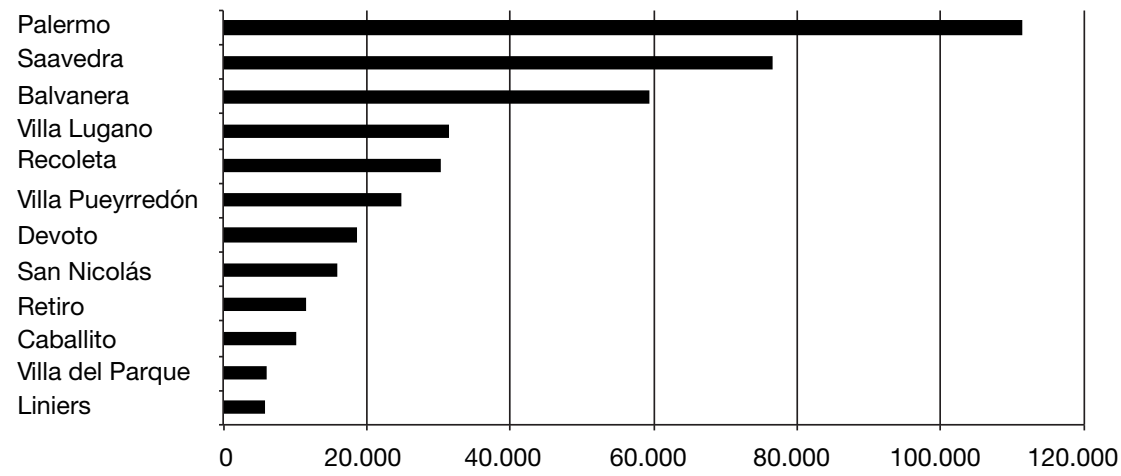

Gráfico 1. SBA (m2) de los centros comerciales de la Ciudad Autónoma de Buenos Aires por barrios (mayo de 2014).

Fuente: elaboración de la autora en base a datos propios y de la Cámara Argentina de Shopping Centers.

$5.900 \mathrm{~m}^{2}$ de SBA, respectivamente, y con un centro comercial cada uno de ellos. El más pequeño en cuanto a superficie total $\left(2.760 \mathrm{~m}^{2}\right)$ y a SBA $\left(1.820 \mathrm{~m}^{2}\right)$ es Galería Güemes. Los barrios de Villa Lugano y Villa Pueyrredón, con un solo centro comercial, ocupan el cuarto y el sexto lugares en orden descendente sobre el total de la ciudad (gráfico 1). Esto se explica porque ambos están formados por galerías comerciales asociadas a una locomotora como son los hipermercados Jumbo y Wall Mart, que demandan una gran cantidad de superficie.

$\mathrm{Al}$ analizar la composición interna de la SBA por barrios, el 49,6\% está destinado a locales comerciales (tabla 6), lo que muestra que, en estos centros comerciales urbanos, desciende la ocupación superficial del sector alimentación - hipermercados con el $25,4 \%$ de la SBA total-y adquiere mayor relevancia el de moda, complementos y hogar. Muy alejado de esas cifras, la SBA destinada a cines ocupa el tercer lugar, con casi un 10\% concentrado en nueve centros comerciales, seguidos de juegos y entretenimientos, con un $7,05 \%$, repartidos en once centros comerciales. Respecto a la superficie de los cines, Village Caballito y Recoleta Mall, especializados en ellos, hacen que se incremente la superficie de esta actividad respecto al total. La SBA de almacenes por departamentos y home centers no tiene ninguna relevancia, por el alto precio del metro cuadrado del suelo. Los dos centros comerciales que disponen de home centers pertenecen a la empresa CENCOSUD. Tienen como proyecto de desarrollo un centro comercial con pocos locales comerciales, basado en un hipermercado Jumbo y un home center como el Easy. Por ejemplo: Portal Palermo tiene una SBA de $32.252 \mathrm{~m}^{2}$, de los cuales solo 4.984 corresponden a locales comerciales; 18.427 , al hipermercado, y 8.841, al home center. Similares características se aprecian en Walmart y Parque Brown Factory Outlet. 
Tabla 6. Composición de la SBA $\left(\mathrm{m}^{2}\right)$ según tipo de actividad comercial por barrios (mayo de 2014)

\begin{tabular}{|c|c|c|c|c|c|c|c|c|c|}
\hline Barrios & № C. C. & Sup. total & $\mathrm{SBA}\left(\mathrm{m}^{2}\right)$ & $\begin{array}{l}\text { SBA }\left(m^{2}\right) \\
\text { locales } \\
\text { comerciales }\end{array}$ & $\begin{array}{c}\mathrm{SBA}\left(\mathrm{m}^{2}\right) \\
\text { cines }\end{array}$ & $\begin{array}{c}\text { SBA }\left(m^{2}\right) \\
\text { juegos y } \\
\text { entretenimientos }\end{array}$ & $\begin{array}{l}\mathrm{SBA}\left(\mathrm{m}^{2}\right) \\
\text { hiperm. / } \\
\text { super. }\end{array}$ & $\begin{array}{l}\mathrm{SBA}\left(\mathrm{m}^{2}\right) \\
\text { home } \\
\text { center }\end{array}$ & $\begin{array}{c}\mathrm{SBA}\left(\mathrm{m}^{2}\right) \\
\text { Tienda } \\
\text { departamental }\end{array}$ \\
\hline Liniers & 1 & $18.700,00$ & $5.800,00$ & $3.700,00$ & 800,00 & $s / d$ & 0 & 0 & $1.300,00$ \\
\hline Villa del Parque & 1 & $18.000,00$ & $5.956,00$ & $1.680,00$ & $1.740,00$ & 590,00 & 0 & 0 & 0 \\
\hline Caballito & 2 & $25.802,00$ & $10.082,31$ & $5.239,31$ & $4.243,00$ & 600,00 & 0 & 0 & 0 \\
\hline Retiro & 1 & $28.982,61$ & $11.615,36$ & $7.907,71$ & $1.601,88$ & $1.444,77$ & 0 & 0 & 661,00 \\
\hline San Nicolás & 2 & $33.549,00$ & $15.811,00$ & $15.781,00$ & 0 & 30,00 & 0 & 0 & 0 \\
\hline Devoto & 1 & $31.296,00$ & $18.741,00$ & $9.048,00$ & $3.808,00$ & $1.570,00$ & $4.315,00$ & 0 & 0 \\
\hline Villa Pueyrredón & 1 & $58.300,00$ & $24.743,00$ & $2.743,00$ & 0 & $10.000,00$ & $12.000,00$ & 0 & 0 \\
\hline Recoleta & 2 & $67.949,54$ & $30.275,80$ & $21.476,76$ & $7.828,64$ & 970,40 & 0,00 & 0 & 0 \\
\hline Villa Lugano & 1 & $60.061,00$ & $31.468,00$ & $10.570,00$ & 0 & 0 & $11.468,00$ & $9.430,00$ & 0 \\
\hline Balvanera & 2 & $160.917,51$ & $59.370,72$ & $33.730,00$ & $9.890,00$ & $9.917,68$ & $4.935,00$ & 0 & 0 \\
\hline Saavedra & 1 & $150.000,00$ & $76.660,00$ & $48.000,00$ & $6.500,00$ & 660,00 & $12.600,00$ & 0 & $8.900,00$ \\
\hline Palermo & 4 & $225.208,53$ & $111.329,16$ & $39.455,83$ & $2.139,68$ & $2.567,00$ & $57.077,20$ & $8.841,00$ & 0 \\
\hline Total & 19 & $878.766,19$ & $401.852,35$ & $199.331,61$ & $38.551,20$ & $28.349,85$ & $102.395,20$ & $18.271,00$ & $10.861,00$ \\
\hline$\%$ & & & 100 & 49,60 & 9,59 & 7,05 & 25,48 & 4,55 & 2,70 \\
\hline
\end{tabular}

Fuente: elaboración de la autora en base a datos propios y de la Cámara Argentina de Shopping Centers.

El barrio de Balvanera registra la mayor SBA destinada a cines, entretenimientos y juegos por la presencia del Abasto Shopping, el segundo centro comercial más grande de la ciudad. En el lado opuesto, el barrio de San Nicolás, en pleno casco histórico y sobre la calle peatonal Florida, dos antiguas galerías se han transformado hoy en sendos centros comerciales (tipología de galería comercial): Galería Pacífico y Galería Güemes. Ninguna de ellas posee hipermercado o supermercado, cines o aparcamiento en el mismo edificio.

Otros datos que describen las características estructurales de los centros comerciales son las plazas de aparcamiento, el número de locales comerciales, las escaleras mecánicas, los ascensores y los visitantes. Los diecinueve centros comerciales de la ciudad disponen de 14.270 plazas de aparcamiento, 2.058 locales comerciales, incluyendo los de restauración, 165 escaleras mecánicas, 66 ascensores y reciben mensualmente 11.240.000 visitantes.

El centro comercial tipo de la Ciudad Autónoma de Buenos Aires tiene una superficie total media de $46.250,85 \mathrm{~m}^{2}$, una SBA media de $21.150\left(\mathrm{~m}^{2}\right), 751$ plazas de aparcamiento, 108 locales comerciales, 3,4 ascensores, 8,7 escaleras mecánicas y una media mensual de 591.579 visitantes. La comparación por barrios, al igual que comentamos con anterioridad, no muestra diferencias considerables, sino que éstas están determinadas más bien por las características de cada centro comercial. Sin embargo, hay algunos aspectos interesantes. El barrio de Palermo, con el mayor número de centros comerciales, de superficie total construida y de SBA, también concentra la cantidad más elevada de plazas de aparcamiento. La relación no es lineal. En el barrio de Villa Lugano, con un solo centro comercial, Parque Brown Factory Outlet, la relación de plazas de aparcamiento es sensiblemente 
Tabla 7. Estructura de los centros comerciales por barrios (mayo de 2014)

\begin{tabular}{|c|c|c|c|c|c|c|}
\hline Barrios & C. C. & $\begin{array}{c}\text { Plazas de } \\
\text { aparcamiento }\end{array}$ & $\begin{array}{l}\text { Locales } \\
\text { comerciales * }\end{array}$ & $\begin{array}{c}\text { Escaleras } \\
\text { mecánicas }\end{array}$ & Ascensores & $\begin{array}{c}\text { Volumen mensual } \\
\text { de visitantes }\end{array}$ \\
\hline Devoto & 1 & 791 & 128 & 10 & 4 & 435.000 \\
\hline Liniers & 1 & 180 & 72 & 2 & 3 & 600.000 \\
\hline Retiro & 1 & 217 & 109 & 9 & 9 & 338.000 \\
\hline Saavedra & 1 & 2.635 & 193 & 22 & 6 & 919.000 \\
\hline Villa del Parque & 1 & 232 & 115 & 4 & 2 & 400.000 \\
\hline Villa Lugano & 1 & 2.452 & 120 & 2 & 0 & 800.000 \\
\hline Villa Pueyrredón & 1 & 1.504 & 22 & 0 & 0 & 250.000 \\
\hline Balvanera & 2 & 1.676 & 279 & 28 & 10 & 2.040 .000 \\
\hline Caballito & 2 & 126 & 98 & 16 & 6 & 990.000 \\
\hline Recoleta** & 2 & 596 & 159 & 21 & 9 & 550.000 \\
\hline San Nicolás & 2 & 0 & 231 & 7 & 4 & 1.180 .000 \\
\hline Palermo & 4 & 3.861 & 532 & 44 & 13 & 2.738 .000 \\
\hline Total & 19 & 14.270 & 2.058 & 165 & 66 & 11.240 .000 \\
\hline
\end{tabular}

Fuente: elaboración de la autora en base a datos propios y de la Cámara Argentina de Shopping Centers.

superior. En menor medida, pero en circunstancias similares, está el Walmart de Villa Pueyrredón. Ambos casos están localizados en zonas periféricas de la ciudad, donde la disponibilidad de suelo es mayor y más barato, lo que permite que todo el complejo tenga una sola planta (por ello, no disponen de ascensores y solo de dos escaleras mecánicas). Y por ser galerías comerciales fundamentadas en un hipermercado, son los que menos locales comerciales poseen.

En el caso de Buenos Aires, el número total de ascensores y escaleras mecánicas por centro comercial está relacionado con tres circunstancias: la construcción en altura por falta de superficie, el alto nivel económico y arquitectónico con el que fue edificado y sus grandes dimensiones. En el primer caso, como ejemplo, Devoto Shopping, con una SBA de $18.741 \mathrm{~m}^{2}$, tiene diez escaleras mecánicas y cuatro ascensores. En el segundo, Patio Bullrich, con nueve ascensores y nueve escaleras mecánicas, y, por sus grandes dimensiones, Abasto Shopping, con veintiuna escaleras mecánicas y siete ascensores.

La mayor afluencia de visitantes por barrios se registra en Palermo, Balvanera y San Nicolás, en ese orden. El centro comercial con mayor volumen de visitantes mensuales es Abasto Shopping (1.740.000), seguido de Alto Palermo Shopping (1.360.000) y Dot Baires (919.000).

Por tanto, los centros comerciales en Argentina han tenido un gran éxito entre los consumidores y han cambiado sensiblemente las prácticas y las motivaciones de consumo de los ciudadanos, como afirma también García Ballesteros (2001) en el caso de España: "han sido un elemento básico en la transformación de las estructuras urbanas y de las prácticas comerciales de sus habitantes». Los datos cuantitativos anteriormente presentados están en total concordancia con las entrevistas realizadas. Las motivaciones respecto al uso de 
los centros comerciales entre los ciudadanos de Buenos Aires son variadas, pero están orientadas básicamente al paseo recreacional y social, a la compra especializada de marcas, al disfrute del cine y de los servicios de restauración, entre otros. De las 216 entrevistas realizadas a pie de calle en los alrededores de los centros comerciales durante el segundo semestre de 2010 , un $58 \%$ se hicieron a mujeres y un $42 \%$, a hombres. Por grupos de edad, el $15 \%$ lo constituían menores de 20 años (entre 15 y 20 años); el 34\% tenía entre 30 y 40 años; el $37 \%$, entre 40 y 60 años, y el 14\% estaba formado por mayores de 60 años.

Los atributos más valorados están estrechamente relacionados con las características inversas a las encontradas en las calles de la ciudad, como seguridad, limpieza, limpieza en los servicios, climatización y horarios. En menor medida, encontramos estar rodeado de gente de la misma clase social, tener buen acceso y aparcamiento. El centro comercial es visto como un lugar de compra, reunión y entretenimiento, y concentra todo lo necesario para la vida diaria, tanto material como inmaterial, además de brindar al consumidor un ambiente agradable, seguro, limpio y climatizado (Cornejo Portugal, 2007):

Sanitarios limpios, sin agresión sonora. Tranquilidad. (Mujer, 60 años, Palermo)

Seguridad, limpieza, horario comercial, climatización, estar con gente de su misma clase social. (Mujer, 32 años, Vicente López)

Fundamentalmente, por seguridad. No me gusta andar con muchas bolsas por la calle, sobre todo cuando son las fiestas. (Mujer, 58 años, Belgrano)

Ninguno de los entrevistados es fiel a los centros comerciales a la hora de comprar, ya que lo hacen tanto allí como en tiendas de la calle, pero lo cierto es que recorren diversos de ellos, pasean, compran, juegan, etc. en sus dependencias, con lo cual realizan un verdadero consumo simbólico al margen del consumo mercantil que se ejerce en su interior (Cornejo Portugal, 2007). A la pregunta «¿Compra siempre en el centro comercial?», han respondido:

No soy fiel a la hora de comprar en un centro comercial, porque depende si alguno me seduce por una oferta, o si tiene una marca que quiero y que otro no tiene. (Mujer, 47 años, Núnez)

No prefiero el shopping, solo si se encuentra a buen precio o debo hacer un regalo. (Mujer, 35 años, Monserrat)

Los entrevistados han confirmado que visitan los centros comerciales desde que aparecieron en la ciudad, lo que demuestra la aceptación inmediata por parte de la ciudadanía. A la pregunta "¿Desde qué fecha visita los shoppings?», han respondido:

Desde los años noventa, que fue el auge de los mismos. (Mujer, 37 años, Caballito)

Desde que se inauguró. (Mujer, 54 años, Avellaneda) 
Claro que también están los detractores de estos espacios, justamente porque los ven como centros de consumo, alienación, destinados a un grupo social al que no pertenecen, o simplemente porque son caros o no les gusta.

No me gusta la ideología del consumismo, detesto el amontonamiento de gente, la oferta sistemática de bienes, me siento encerrada. (Mujer, 45 años, Almagro)

No me gustan porque prefiero el aire libre. Los shoppings son cerrados, ruidosos y sin luz natural. Conozco varios: Paseo Alcorta, Alto Palermo, Unicenter. (Mujer, 49 años, Villa Urquiza)

A lo largo del trabajo, se ha recalcado que la mayoría de centros comerciales de Buenos Aires están orientados hacia sectores socioeconómicos medios altos y altos, y esto depende de su localización, como, por ejemplo: Patio Bullrich, Paseo Alcorta, Galerías Pacífico y Unicenter, de la zona norte:

Gente de sectores de ingresos altos y medios altos (burguesía y pequeña burguesía, en otros términos), que compran compulsivamente y gustan de ocupar tardes enteras en dicha actividad, gastando mucho más de lo que cuestan los mismos productos (o mejores) en tiendas comunes. También concurre gente de otras clases sociales, aunque en menor medida, que pretenden aproximarse y/o aparentar pertenecer a esos sectores. (Mujer, 39 años, Parque Avellaneda)

Algunos entrevistados tienen la percepción de que, en años anteriores, los centros comerciales eran lugares de distinción social orientados a un público de alto poder adquisitivo: «Sí, creo que antes era más pronunciado. Hoy ya no, igualmente algunos más que otros» (hombre, 34 años, Santos Lugares). Esta apreciación es real. En los últimos años, se ha atravesado por un nuevo boom del consumo, no solo de los sectores medios altos y altos, sino y fundamentalmente, por los sectores medios y medios bajos, que aspiran a un nivel superior, lo que en parte creen lograr consumiendo en los centros comerciales.

En los últimos años, este sector empresarial tiende a cubrir las necesidades de consumo de la base social de la población (sectores medios bajos), mediante la apertura de centros comerciales orientados claramente a estos niveles, lo que podría tener una doble interpretación: por un lado, cubrir un mercado insatisfecho en zonas vírgenes de centros comerciales (racionalidad mercantil) y, por otro, generar espacios destinados a esos sectores, con precios adaptados a su poder adquisitivo, lo que «impide» implícitamente que consuman o visiten los centros comerciales orientados a sectores sociales más exclusivos. Por tanto, se estarían construyendo o generando espacios de consumo capaces de atraer a personas que se identifican entre sí, sin dejar fuera a casi nadie del placer de consumir en un centro comercial.

\section{Conclusión}

Como hemos visto en los párrafos precedentes, el ritmo de crecimiento de los centros comerciales siempre ha sido positivo y, hasta el momento, no se ha 
cerrado ninguno de ellos. Desde las primeras aperturas, se logra, en pocos años, una expansión muy acelerada, tanto de centros comerciales como de superficie construida. La desaceleración económica de fines de la década de 1990 hace eclosión en diciembre de 2001, lo cual paraliza la actividad en el sector. La recuperación de la economía nacional y los altos niveles de consumo de la sociedad en general permitieron el rápido crecimiento del sector.

En mayo de 2014, se contabilizan 113 centros comerciales en todo el país, con una SBA de $1.966 .826,51 \mathrm{~m}^{2}$, una dimensión media de $23.139,14 \mathrm{~m}^{2}$ de SBA, una densidad comercial media de $50,38 \mathrm{~m}^{2}$ de SBA cada 1.000 habitantes, 42.759 .500 de visitantes mensuales, 94.974 plazas de aparcamiento y un $58,9 \%$ de los centros comerciales que poseen salas de cine. Estos son algunos de los indicadores que caracterizan a los shopping centers y que evidencian la expansión del sector en este país.

En general, la tendencia en Argentina es decantarse por la apertura de centros comerciales de menor tamaño y con una oferta más diversificada. El número de centros comerciales está creciendo sustancialmente en los últimos años por la apertura en ciudades más pequeñas y aún sin conquistar por este formato comercial. El resto del país es un área de gran desarrollo potencial. Tenemos contabilizados dieciocho proyectos en marcha, algunos en construcción y otros planeados.

La concentración en el Aglomerado Gran Buenos Aires está estrechamente relacionada con la alta densidad de población y, aún, con el mediano y alto nivel socioeconómico. La elevada densidad comercial es mucho mayor en la Ciudad Autónoma de Buenos Aires respecto a los partidos del Aglomerado y aún más en comparación con el resto del país. Este indicador apoya la tesis de que los centros comerciales argentinos tienden a localizarse en la trama urbana muy consolidada frente a la suburbana. Los centros comerciales en Buenos Aires refuerzan la centralidad comercial y urbana y aún no son gérmenes de nuevas centralidades, aunque esta tendencia podría modificarse en los próximos años, como vemos con el incremento de la SBA y de aperturas de centros comerciales en los suburbios del Gran Buenos Aires y del resto del país. Aún es una industria poco desarrollada en Argentina, puesto que quedan muchas áreas vírgenes por cubrir.

Los centros comerciales en Buenos Aires presentan rasgos originales, características muy localistas que los diferencian de sus modelos de referencia, como muestran las encuestas realizadas en la capital sobre la motivación del uso que se hace de ellos. Demuestran la incorporación social del nuevo formato a la vida cotidiana desde los comienzos de la actividad, su utilización frecuente, el desplazamiento hacia muchos de ellos para conocerlos o consumir en sus dependencias y el hacerlos parte de la vida diaria como lugar de encuentro social. Los centros comerciales son percibidos como ambientes seguros, limpios, con variedad de ofertas y amplios horarios, y se los destina básicamente al paseo recreacional y social, a la compra especializada de marcas, al disfrute del cine y, fundamentalmente, al uso de los servicios de restauración (el ir a tomar el "café» porteño), entre otros. 
La falta de una base de datos completa sobre todos los centros comerciales del país ha dificultado el análisis de los mismos, por lo cual queda planteada la tarea de centralización y organización para un futuro próximo, ya sea como colaboración con la Cámara Argentina de Shopping Centers o con algún otro organismo similar.

No podemos negar lo que es una realidad, pero sí podemos cuestionarla, repensarla, hacer nuevas formas en y de la ciudad, pero, para ello, debemos partir de un análisis empírico, ver el comportamiento general que muestran dicho fenómeno y la sociedad en su conjunto.

\section{Referencias bibliográficas}

Amendola, Giandomenico (2000). La ciudad postmoderna. Madrid: Celeste Ediciones. APSA (1998). Alto Palermo: Memoria y estado contable. Buenos Aires: APSA.

- (2004). Alto Palermo: Memoria y estado contable. Buenos Aires: APSA.

- (2008). Alto Palermo: Memoria y estado contable. Buenos Aires: APSA.

BlaKsley, Santiago (2011). "La industria de los shopping centers cumple 25 años». IV Congreso Internacional de Shopping Centers, 21-22 de septiembre. Buenos Aires.

Caprón, Guenola (1996). La ville privée: Les shopping centers à Buenos Aires. Universidad de Toulouse II. Tesis doctoral.

Cornejo Portugal, Inés (2007). El lugar de los encuentros. México: Universidad Iberoamericana.

Equipo de Gestión Económica y Social, Ciudad Autónoma de Buenos Aires (2009).

<www.eges.com.ar/archivos/informes/1258722597_informe-indicadorescaba-2009.pdf> [Consulta: 5 enero 2014].

Escudero Gómez, Luis (2008). Los centros comerciales. Cuenca: Universidad de Castilla La Mancha.

Featherstone, Mike (2000). Cultura de consumo y posmodernismo. Buenos Aires: Amorrortu.

Ferreira Freitas, Ricardo (1996). Centres commerciaux: Îles urbaines de la post-modernité. París: L'Harmattan.

García Ballesteros, Aurora (2001). «Las motivaciones hacia el uso de los centros comerciales en Madrid». Anales de Geografía, 21, 257-284.

García Escalona, Emilia (1997). «Espacio central y actividad comercial». Distribución y Consumo, junio-julio, 11-21.

Grossman, Luis (1988). «Sobre los shopping centers». Summa de Arquitectura, 256, diciembre, 22-32.

Lana Seabra, Raphael y Marvulle Bueno, Fabio (2012). «El protagonismo brasileño en el siglo xxI: ¿Subimperialismo o semiperiferia? Rebela, 2 (1), junio, 75-86.

Leston, Eduardo (28 de noviembre 1992). «El fenómeno shopping center». Clarín. Suplemento «Arquitectura, Ingeniería, Planeamiento y Diseño», 2 y 3.

Osuna Gutierrez, Patricia (9 de septiembre 2013). «La expansión que no cesa». La Nación. Sección «Inmuebles Comerciales».

Padilha, Valquíria (2006). Shopping Center. San Pablo: Boitempo.

Pintaudi, Silvana (1989). O templo da mercadoria. San Pablo: Universidad de San Pablo. Tesis doctoral. 
Santos, Milton (1987). O espaço do cidadao. San Pablo: Nobel.

SARLO, Beatriz (1996). "La ciudad y los shoppings: Una visión crítica». Revista de Arquitectura, 64-70, julio-octubre. Sociedad Central de Arquitectos.

- (2000). Escenas de la vida posmoderna. Buenos Aires: Ariel.

Sassano Luiz, Silvana (2001). "Transformación de un espacio urbano: El caso del Mercado de Abasto de Buenos Aires». Anales de Geografia, 21, 99-118. Universidad Complutense.

Soja, Edward (1996). Thirdspace: journeys to Los Angeles and other real-and-imagined places. Cambridge: Blackwell.

- (2008). Postmetrópolis. Madrid: Traficantes de Sueños.

Tella, Guillermo (2005). «Rupturas y continuidades en el sistema de centralidades de Buenos Aires». En: Welch Guerra, Max (edit.). Buenos Aires a la deriva. Buenos Aires: Biblos.

- (2007). Un crack en la ciudad. Buenos Aires: Nobuko.

Wallerstein, Immanuel (1979). The Capitalist World-Economy. Londres: Cambrigde University Press.

\section{Bibliografia web}

Asociación Española de Centros Comerciales (2013). <http://www.aedecc.com>. [Consulta: 27 noviembre 2013].

Cámara Argentina de Shopping Centers (2014). <www.casc.org.ar> [Consulta: 9 mayo 2014].

ObSERVATORIO InMOBILIARIo y de LA CONSTRUCCión. <www.observatorioinmobiliario.es> [Consulta: 2 noviembre 2011].

\section{Entrevistas}

Baum, Silvina (gerente de marketing de Village Recoleta y Recoleta Mall). Entrevista realizada el 25 de septiembre de 2007 en la ciudad de Buenos Aires. Entrevistadora: Silvana Sassano.

Goilenberg, Eduardo (director de Nuevos Negocios, Alto Palermo, APSA). Entrevista realizada el 29 de agosto de 2002 en la ciudad de Buenos Aires. Entrevistadora: Silvana Sassano.

LípARe, Alberto (ex gerente de la Cámara Argentina de Shopping Centers). Entrevista realizada el 16 de agosto de 2001 en la ciudad de Buenos Aires. Entrevistadora: Silvana Sassano.

PÉrez Arias, Mónica (gerente general de la Cámara Argentina de Shopping Centers). Entrevista realizada el 17 de septiembre de 2007 en la ciudad de Buenos Aires. Entrevistadora: Silvana Sassano. 